\title{
¿Solicitan las mujeres más consultas al área médica que los hombres?
}

\author{
F. ROSA-JIMÉNEZ, A. M. MONTIJANO CABRERA, C. ÍLIA HERRÁIZ \\ MONTALVO $^{1}$, J. L. ZAMBRANA GARCÍA
}

\author{
Líneas de Procesos Generales del Adulto y Área de Sistemas de Información. Hospital \\ Alto Guadalquivir. Andújar. Jaén. ${ }^{1}$ Hospital de Montilla. Montilla. Córdoba.
}

\author{
DO WOMEN DEMAND TO BE ASSISTED AT MEDICAL CLINICS \\ MORE OFTEN THAN MEN?
}

\section{RESUMEN}

Introducción: Las peculiaridades de los usuarios del sistema sanitario público español han sido evaluadas en sus distintos aspectos, sobre todo, en atención primaria y urgencias. Sin embargo, las características epidemiológicas de los pacientes que acuden a las consultas externas de las distintas especialidades lo han sido menos.

Pacientes y método: Se analizó la procedencia, el origen, la edad y el sexo de todas las solicitudes de primera cita para las consultas externas del área médica de nuestro hospital (medicina interna, cardiología, digestivo y neumología) durante el año 2002.

Resultados: El 53,4\% de las citas fueron generadas por mujeres que representaron el $53,8 \%$ de la población demandante. Estas solicitaron más citas que los varones con relación a la población de referencia en atención primaria, en el medio urbano y en los pacientes menores de 60 años. El área urbana generó un porcentaje de citas superior a la del área rural. No se observó un mayor índice de frecuentación entre las mujeres respecto a los varones pero sí en los pacientes procedentes del medio urbano respecto al medio rural.

Conclusiones: En nuestra área de referencia se observa una mayor demanda sanitaria de la población femenina, aunque ésta va a depender de factores tales como la edad, la localidad de procedencia y el origen de la solicitud de asistencia.

PALABRAS CLAVE: Demanda asistencial. Asistencia ambulatoria. Epidemiología. Derivación de pacientes. Atención hospitalaria.

\section{ABSTRACT}

Background: Different features of the users of the Spanish Public Health System have been previously assessed, specially in General Practice and Hospital Emergency Departments. Nevertheless, the epidemiological characteristics of those patients who attend to specialised clinics have not been so thoroughly evaluated.

Patients and methods: The referee of the demand, the place of residence, the age and the sex of patients were all analysed for the, first visits, at the Medical Department clinics (Internal Medicine, Cardiology, Gastroenterology, Pneumology) in our hospital, during 2002.

Results: A total of 7,486 demands for attention (53.3\% were women) were asked for by 5,841 patients (52.8\%) were women. When analysing the variable , place of residence, we find women asked for more frequently than men, in the categories of General Practice Department and age less than 60 years. Those patients coming from urban areas were more in percentage than those coming from rural areas. Whereas the, index of frequency, was higher in women than in men, however, there were no differences between urban and rural areas.

Conclusion: In our targeted population we have observed a higher demand for medical clinics in women, though the actual demand will depend on factors such as age, place of residence and the referee.

KEY WORDS: Demand for attendance. Outpatient assistance. Epidemiology. Referral of patients. Hospital attendance.

Rosa-Jiménez F, Montijano Cabrera AM, Ília Herráiz. Montalvo C, Zambrana García JL. ¿ Solicitan las mujeres más consultas al área médica que los hombres? An Med Interna (Madrid) 2005; 22: 515-519.

\section{INTRODUCCIÓN}

Las características de los pacientes que acuden al sistema sanitario público español han sido el centro de atención de numerosas publicaciones. Se han evaluado las características geográficas (núcleos rurales, zonas urbanas, distancias al centro sanitario) (1); las diferencias sociodemográficas (nivel cultural, situación laboral, problemática social, estructura familiar) (2); los motivos de consulta (patrones de morbilidad, patologías crónicas); los tipos de asistencia (programada, urgente, domiciliaria); los servicios sanitarios (estructura y organización, competencia del facultativo, grado de confianza) (3); las fluctuaciones temporales (climatología, día de la semana, franjas horarias); las particularidades del paciente (estado de salud, hiperfrecuentación, percepción de enfermedad, expectativas) (4), e, incluso, la influencia de determinados eventos (retransmisiones deportivas, mercados ambulantes, contaminación atmosférica) (5). Dichos estudios han 
tenido como campo de trabajo las consultas de atención primaria o las áreas de urgencias hospitalarias $(6,7)$ reflejando con ello los rasgos de los usuarios que demandan asistencia sanitaria en el primer nivel asistencial. Sin embargo, el perfil del paciente que utiliza el segundo escalón sanitario (consultas externas hospitalarias y centros periféricos de especialidades) ha sido menos valorado. Se ha analizado la calidad de los documentos de derivación hospitalaria, la concordancia diagnóstica, los motivos de derivación más frecuentes o la mayor o menor adecuación de las mismas (8-10) pero son escasos los trabajos que analizan las características epidemiológicas o geográficas y que tengan en consideración la estructura poblacional del área evaluada.

El presente estudio tiene como objetivo analizar si la demanda sanitaria al área médica de nuestro hospital sigue algún patrón epidemiológico (edad y sexo) relacionado con el tipo de asistencia solicitada (pacientes derivados por atención primaria respecto a los pacientes remitidos desde urgencias) o su localización geográfica (residentes en un núcleo urbano respecto de los habitantes procedentes del ámbito rural).

\section{PACIENTES Y MÉTODO}

La empresa pública "Hospital Alto Guadalquivir" es un centro hospitalario público de primer nivel ubicado en la población jiennense de Andújar que ofrece cobertura sanitaria a algo más de 65.000 habitantes. Poco más de la mitad de la población se concentra en dicha localidad mientras que el resto se reparte de forma heterogénea en los 7 municipios que conforman su área de referencia. La asistencia especializada ambulatoria se realiza en el bloque de consultas externas, sito dentro del propio hospital.

Se analizaron los datos epidemiológicos (localidad de procedencia, origen de la consulta, edad y sexo) de todas las solicitudes de primera cita para las consultas externas del área médica (medicina interna, cardiología, digestivo y neumología) durante el año 2002. Se consideraron como tales: 1) los documentos de derivación hospitalaria normalizados enviados por los facultativos de atención primaria; 2) las citas generadas por el área de urgencias de nuestro hospital; y 3) las interconsultas solicitadas por cualquier especialidad de nuestro hospital. No se incluyeron los pacientes citados exclusivamente para la realización de pruebas complementarias (pruebas funcionales respiratorias, fibrobroncoscopia, ecocardiografía, ergometría, electrocardiograma, holter 24 horas, endoscopia oral o colonoscopia) ni las segundas visitas programadas directamente en consulta externa (revisiones). Se excluyeron los pacientes ajenos a nuestra área de referencia, las interconsultas procedentes de otros centros hospitalarios y todos los menores de 15 años. Los datos poblacionales fueron obtenidos del padrón municipal de fecha 1 de enero de 2002 publicado por el Instituto Nacional de Estadística (INE). Dicho documento presenta los grupos de edad agrupados en quinquenios. Así, los pacientes de 14 años están incluidos en el grupo de 10-14 años (que incluye pacientes en edad pediátrica), razón por la cual fueron excluidos del estudio. El resto de pacientes fueron agrupados en 5 grupos: de 15 a 29 años, de 30 a 44 años, de 45 a 59 años, de 60 a 74 años y de edad igual o superior a 75 años.

En función del domicilio del paciente se estableció una variable dicotómica: área urbana (domiciliados en Andújar) y área rural (procedentes del resto de localidades de la comarca). Se calcularon distintos porcentajes (número de citas x 100 / población seleccionada) en función del sexo de los pacientes, el grupo de edad, la zona geográfica de procedencia y el origen de la solicitud de consulta. Se consideró índice de frecuentación la media ( \pm una desviación estándar) del número de citas solicitadas por cada uno de los pacientes incluidos en el estudio y se expresó como citas/paciente. El estudio estadístico se realizó con el programa SPSS 9.0 ${ }^{\circledR}$. Se utilizó el test de Student para la comparación de medias procedentes de muestras independientes y el test de la c2 para la comparación de variables dicotómicas.

\section{RESULTADOS}

\section{POBLACIÓN DE REFERENCIA}

De los 65.124 habitantes de nuestra área de referencia, el $18,1 \%$ (11.813) tenían menos de 15 años. Los 53.311 restantes formaron parte del análisis estadístico: el 58,2\% (31.040) vivían en Andújar (área urbana) y el restante 41,8\% (22.271) en las distintas localidades de la comarca (área rural). El $50,9 \%$ (27.149) eran del sexo femenino frente al $49,1 \%$ (26.162) de varones. La distribución por grupos de edad y sexo se muestra en la figura 1.

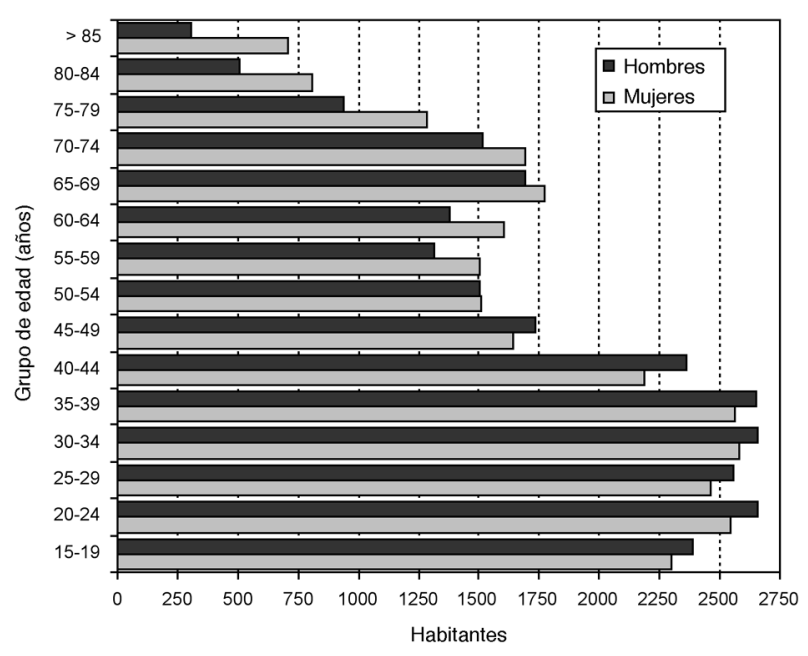

Fig. 1. Estructura poblacional de la comarca de Andújar (año 2002).

\section{SOLICITUDES DE CITAS}

Durante el año 2002 se generaron un total de 7.486 solicitudes de primera visita al área médica de nuestro hospital. Se excluyeron 426 (5,7\%): 111 de localidades fuera del área de referencia, 75 de pacientes menores de 15 años, 4 por falta de datos y 236 por citas duplicadas. Las restantes 7.060 (94,3\%) procedían de un total de 5.841 pacientes, de los cuales el $53,8 \%$ eran mujeres (3.140) y el $46,2 \%$ varones (2.701). El $53,4 \%$ de las citas (3.770) fueron generadas por pacientes del sexo femenino frente a un 46,6\% (3.290) por varones. El $78,7 \%$ de las citas (5.555) procedían de atención primaria, el 
TABLA I

PORCENTAJE DE CITAS EN FUNCIÓN DEL SEXO

\begin{tabular}{|c|c|c|c|c|c|c|c|c|c|c|}
\hline GRUPOS & Citas & $\begin{array}{c}\text { Global } \\
\text { Población }\end{array}$ & $\%$ & Citas & $\begin{array}{c}\text { Mujeres } \\
\text { Población }\end{array}$ & $\%$ & Citas & $\begin{array}{l}\text { Hombres } \\
\text { Población }\end{array}$ & $\%$ & $p^{*}$ \\
\hline Total de citas & 7.060 & 53.311 & 13,2 & 3.770 & 27.149 & 13,9 & 3.290 & 26.162 & 12,6 & $<0,001$ \\
\hline Atención primaria & 5.555 & 53.311 & 10,4 & 3.036 & 27.149 & 11,2 & 2.519 & 26.162 & 9,6 & $<0,001$ \\
\hline Urgencias & 1.024 & 53.311 & 1,9 & 482 & 27.149 & 1,8 & 542 & 26.162 & 2,1 & $<0,05$ \\
\hline Interconsultas & 481 & 53.311 & 0,90 & 252 & 27.149 & 0,93 & 229 & 26.162 & 0,87 & n.s. \\
\hline Núcleo urbano & 4.649 & 31.040 & 15 & 2.520 & 15.922 & 15,8 & 2.129 & 15.118 & 14,1 & $<0,001$ \\
\hline Zona rural & 2.411 & 22.271 & 10,8 & 1.250 & 11.227 & 11,1 & 1.161 & 11.044 & 10,5 & n.s. \\
\hline $15-29$ años & 709 & 14.904 & 4,8 & 414 & 7.309 & 5,7 & 295 & 7.595 & 3,9 & $<0,001$ \\
\hline 30-44 años & 1.199 & 14.990 & 8 & 637 & 7.321 & 8,7 & 562 & 7.669 & 7,3 & $<0,01$ \\
\hline 45-59 años & 1.352 & 9.116 & 14,8 & 736 & 4.660 & 15,8 & 616 & 4.456 & 13,8 & $<0,01$ \\
\hline 60-74 años & 2.442 & 9.649 & 25,3 & 1.265 & 5.063 & 25 & 1.177 & 4.586 & 25,7 & n.s. \\
\hline$\geq 75$ años & 1.358 & 4.552 & 29,8 & 718 & 2.796 & 25,7 & 640 & 1.756 & 36,4 & $<0,001$ \\
\hline
\end{tabular}

$\%$ expresados en citas/100 habitantes; ${ }^{*}$ ) Diferencia entre hombres y mujeres.

$14,5 \%$ (1.024) se originaron en el área hospitalaria de urgencias y el restante $6,8 \%$ (481) fueron interconsultas de distintos servicios hospitalarios. El 44,5\% de las citas (3.141) fueron dirigidas a medicina interna, el 25,9\% (1.826) a digestivo, el $15,3 \%$ (1.080) a cardiología y el restante $14,3 \%$ (1.013) a neumología.

\section{EDAD MEDIA}

En función del sexo, la edad media de los 5841 pacientes no mostró diferencias estadísticamente significativas en el global de los pacientes [57,2 (18,7) años en varones vs. 56,3 $(19,2)$ años en mujeres]; en los derivados desde atención primaria $[57,4(18,6)$ vs. $56,9(19,1)]$; en los procedentes de urgencias $[57,5(19,4)$ vs. 56,2 $(20,2)]$, en los habitantes del área urbana $[55,2(19,3)$ vs. 56,1 $(18,7)]$ ni en los procedentes del área rural $[58,4(18,9)$ vs. $59(18,5)$ años]. Con respecto a las interconsultas, la edad media de los varones fue superior a la de las mujeres [59,2 (16,4) vs. 53,2 (17) años; $\mathrm{p}<0,001]$.

Respecto al entorno geográfico, la edad media de los pacientes procedentes del medio rural fue superior a la del medio urbano [58,7 $(18,7)$ vs. 55,6 $(19,1)$ años; $\mathrm{p}<0,001]$. No se observaron diferencias en la edad media de los pacientes de atención primaria respecto a los procedentes de urgencias [57,1 (18,9) vs. 56,9 (19,8)].

\section{PORCENTAJE DE CITAS: EDAD Y SEXO}

El porcentaje de solicitud de citas con relación a la población de referencia fue superior en las mujeres tanto en el ámbito global como en los pacientes menores de 59 años (Tabla 1). En edades superiores los resultados se invierten, siendo mayor en los varones de edad igual o superior a 75 años. Los valores se incrementaron progresivamente en función de la edad, oscilando entre 4,8 citas/100 adolescentes hasta las 29,8 citas/100 pacientes de más de 75 años (Tabla I).

\begin{tabular}{|c|c|c|c|c|}
\hline \multicolumn{5}{|c|}{ TABLA I } \\
\hline \multicolumn{5}{|c|}{ ÍNDICE DE FRECUENTACIÓN EN FUNCIÓN DEL SEXO } \\
\hline & Global & Mujeres & Hombres & $p^{*}$ \\
\hline Citastotales & $1,21(0,5)$ & $1,2(0,5)$ & $1,22(0,5)$ & ns \\
\hline Núcleo urbano & $1,23(0,5)$ & $1,23(0,5)$ & $1,24(0,6)$ & ns \\
\hline Zona rural & $1,16(0,4)$ & $1,15(0,4)$ & $1,17(0,5)$ & ns \\
\hline 15-29años & $1,11(0,3)$ & $1,12(0,3)$ & $1,1(0,3)$ & ns \\
\hline 30 - 44 años & $1,15(0,4)$ & $1,15(0,4)$ & $1,15(0,4)$ & ns \\
\hline 45 - 59 años & $1,2(0,5)$ & $1,19(0,5)$ & $1,21(0,5)$ & ns \\
\hline 60 - 74 años & $1,26(0,6)$ & $1,26(0,6)$ & $1,25(0,6)$ & ns \\
\hline
\end{tabular}

Valores (citas/paciente) expresados en media (DS). Test de Student. $\left({ }^{*}\right)$ Diferencia entre hombres y mujeres

No se observaron diferencias estadísticamente significativas en las interconsultas.

\section{ATENCIÓN PRIMARIA VS. URGENCIAS HOSPITALARIAS}

La solicitud de citas de las mujeres fue mayor que la de los hombres en las citas originadas desde atención primaria, pero fue menor en las producidas en urgencias (Tabla I). Respecto a la edad, atención primaria sigue la misma tendencia que la población global, siendo superior en las mujeres de entre $15 \mathrm{y}$ 59 años e invirtiéndose en el grupo de 75 años o más (Tabla III). En los pacientes derivados desde urgencias se observa un predominio femenino en edades jóvenes ( $<44$ años) y un predominio masculino en edades avanzadas ( $>60$ años).

\section{ÁREA GEOGRÁFICA}

El área urbana generó más citas que el medio rural. Estos datos se confirmaron en el porcentaje absoluto de citas $(66,8 \%)$, en el porcentaje de citas [15 citas/100 habitantes urbanos vs. 10,8 citas/100 habitantes rurales; $\mathrm{p}<0,001]$, en 
TABLA I

-NÚMERO DE CITAS EN VALORES ABSOLUTOS Y PORCENTAJES AJUSTADOS A LA POBLACIÓN DE REFERENCIA

\begin{tabular}{|c|c|c|c|c|c|c|c|c|c|c|c|c|c|c|c|c|c|c|c|c|}
\hline \multirow{3}{*}{$\begin{array}{l}\text { Edad } \\
\text { Años }\end{array}$} & & & \multirow{2}{*}{\multicolumn{3}{|c|}{$\begin{array}{l}\text { Atención pimaria } \\
\text { Mujeres }\end{array}$}} & \multicolumn{5}{|c|}{ Urgencias } & \multicolumn{5}{|c|}{ Medio Urbano } & \multicolumn{5}{|c|}{ Medio rural } \\
\hline & \multicolumn{2}{|c|}{ Hombres } & & & & \multicolumn{2}{|c|}{ Hombres } & \multicolumn{2}{|c|}{ Mujeres } & \multirow[b]{2}{*}{$p^{*}$} & \multicolumn{2}{|c|}{ Hombres } & \multicolumn{2}{|c|}{ Mujeres } & \multirow[b]{2}{*}{$p^{*}$} & \multicolumn{2}{|c|}{ Hombres } & \multicolumn{2}{|c|}{ Mujeres } & \multirow[b]{2}{*}{$p^{*}$} \\
\hline & Citas & $\%$ & Citas & $\%$ & $p^{*}$ & Citas & $\%$ & Citas & $\%$ & & Citas & $\%$ & Citas & $\%$ & & Citas & $\%$ & Citas & $\%$ & \\
\hline-29 & 236 & 3,1 & 324 & 4,4 & $<0,001$ & 47 & 0,6 & 66 & 0,9 & $<005$ & 204 & 4,4 & 295 & 6,6 & $<0,001$ & 89 & 3 & 119 & 4,1 & $<0,05$ \\
\hline & 110 & 5,3 & & 6,7 & & 19 & 1,5 & 8 & 1,2 & 00 & 378 & 8,6 & & 9,9 & & & 5,5 & & 6,8 & $<0$ \\
\hline $.5-59$ & 482 & 10,8 & 586 & 12,6 & $<0,01$ & 74 & 1,7 & 85 & 1,8 & n.s & 427 & 15,7 & 530 & 18,5 & $<, 0,01$ & 185 & 10,1 & 206 & 11,5 & n.s. \\
\hline $50-74$ & 921 & 20,1 & 1044 & 20,6 & n.s & 176 & 3,8 & 141 & 2,8 & $<0,01$ & 733 & 30 & 804 & 29,3 & & 444 & 20,7 & 461 & 19,9 & n.s. \\
\hline 75 & 470 & 26,8 & 589 & 21,1 & $<0,001$ & 125 & 7,1 & 102 & 3,6 & 0001 & 382 & 43,2 & 453 & 31,1 & $<0,001$ & 258 & 29,6 & 265 & $19,8<$ & $<0,001$ \\
\hline
\end{tabular}

Test de la $\chi^{2} ;\left(^{*}\right)$ Diferencia entre hombres y mujeres.

cada uno de los grupos de edad (Tabla III), en atención primaria $[11,8$ vs. 8,$5 ; \mathrm{p}<0,001]$ y en urgencias $[2,1$ vs. 1,$5 ; \mathrm{p}<$ $0,001]$.

En el área urbana el porcentaje de citas de las mujeres fue superior a la de los varones pero no en el área rural donde fueron similares (Tabla I). Respecto a los grupos de edad, en el área urbana se observó un predominio femenino entre los 15 y 59 años y masculino en la población mayor de 75 años (Tabla III). En el área rural el porcentaje de citas en las mujeres es superior al de los varones entre los 15 y 44 años invirtiéndose los porcentajes en los pacientes de más de 75 años.

\section{ÍNDICE DE FRECUENTACIÓN}

La frecuentación femenina no mostró diferencias estadísticamente significativas respecto a la masculina en el global de citas ni en función de la localización geográfica (Tabla II). Sin embargo sí se observó una mayor frecuentación en los pacientes procedentes del núcleo urbano respecto a los del área rural $[1,23(0,5)$ citas/paciente urbano vs. $1,16(0,4)$ citas/paciente rural; $\mathrm{p}<0,001]$. La frecuentación se incrementó progresivamente con la edad tanto en el ámbito urbano como en el rural $\mathrm{y}$ tanto en hombres como en mujeres.

\section{DISCUSIÓN}

En nuestra serie, las mujeres solicitan más primeras citas que los varones, tanto en número absoluto como en porcentajes ajustados a la población de referencia. No obstante, esta afirmación queda matizada por los resultados en los distintos subgrupos analizados, en los que se observa que las cifras son similares en las derivaciones procedentes del área rural y que existe un predominio masculino en las citas originadas desde urgencias. Algunos autores (11) justifican la mayor presencia de la mujer en las consultas médicas en una peor percepción de éstas de su salud, con una mayor restricción de sus actividades, un mayor número de síntomas, más sensación de dolor y una mayor notificación de enfermedades crónicas que los varones, hecho ya puesto de manifiesto en la última encuesta nacional de salud del Ministerio de Sanidad y Consumo (2003). No se ha demostrado que la superior solicitud de citas del sexo femenino se acompañe de un mayor índice de fre- cuentación, es decir, acuden a las distintas consultas en mayor número que los varones pero el mismo número de veces. Estos resultados estarían en consonancia con la mayor parte de los estudios realizados en el ámbito de la atención primaria (12), aunque otros autores consideran que la edad o el sexo de los pacientes no condicionan las características de la demanda de atención sanitaria y sí sus patologías $(13,14)$. Se ha comprobado la influencia de la edad en relación hacia una mayor proporción de citas en los varones de edad avanzada y de mujeres en edades más jóvenes. Asimismo, se ha comprobado que el índice de frecuentación y el porcentaje de citas han aumentado proporcionalmente con la edad, de forma que el $30 \%$ de la población mayor de 75 años ha acudido al menos una vez a alguna de nuestras consultas frente a menos del 5\% de la población menor de 30 años. Ello confirma el hecho ya conocido de que la edad es una de las variables predictivas más importante en el uso de servicios médicos, siendo los ancianos el grupo etario que genera una mayor demanda asistencia y con ello un mayor consumo de recursos sanitarios (15).

Se han descrito variaciones en la utilización de las consultas de atención primaria en función de su localización en un medio rural o en un entorno urbano (16). La mayor tasa de derivación e índice de frecuentación en el área urbana podría estar con relación a una mayor accesibilidad al nivel hospitalario (17) aunque se han descrito otros factores que atañen al medio rural (mejor conocimiento del medio, mayor prestigio de los médicos de cabecera, elevado poder de persuasión de los mismos, mayor nivel resolutivo de patologías banales, distinta distribución de grupos nosológicos, etc.) (18). El 80\% de las citas solicitadas a nuestra área hospitalaria provenían de atención primaria por lo que es lógico pensar que dichas derivaciones reflejen, de alguna manera, lo que acontece en dicho nivel asistencial. Por el contrario, los resultados procedentes del área de urgencias deben ser analizarlos con cautela ya que sólo representan un pequeño porcentaje del total de urgencias atendidas en nuestro hospital y pueden no ser extrapolables al total de pacientes que solicita asistencia urgente. Nuestros resultados se decantan hacia una mayor tasa de derivación hacia consultas externas de los varones que acuden a urgencias respecto a las mujeres aunque en la literatura se encuentran datos contradictorios tanto a favor de una mayor presencia de consultantes varones en las áreas de urgencias $(18,19)$ como de igualdad entre ambos sexos $(20,21)$. 
Los trabajos que valoran globalmente las derivaciones desde atención primaria hacia las distintas especialidades médicas y quirúrgicas muestran resultados dispares en función a las distintas especialidades analizadas (22-24). Se ha descrito una mayor demanda de asistencia del sexo femenino en las consultas externas de dermatología (25), reumatología (26), psiquiatría (27) y neurología (28); proporciones similares en consultas de traumatología (29) y un predominio masculino en consultas de neumología (30).

\section{Bibliografía}

1. Alfonso Sánchez JL, Sanchis-Bayarri Vaillant V. Atención primaria en el medio rural: estudio comparativo con el medio urbano. Med Clin (Barc) 1986; 86: 143-146.

2. McNiece R, Majjed A. Socieconomic differences in general practice consultation rates in patients aged 65 and over: prospective cohort study. BMJ 1999; 319: 26-28.

3. Llobera Cànaves J. La derivación de pacientes de la atención primaria a la especializada. Gac Sanit 1988; 2: 271-275.

4. Muñoz Baragaño P, Blanco Suárez AM, García Lavandera LJ, Alonso Fernández M, Salvadores Rubio J, Alonso Arias PS. Estudio comparativo entre población normo e hiperfrecuentadora en un centro de salud. Aten Primaria 1996; 18: 484-490

5. Miró O, Sánchez M, Borrás A, Millá J. Fútbol, televisión y servicios de urgencias. Med Clin (Barc) 2000; 114: 538-539.

6. Buitrago Ramírez F, Chávez García M, Del Cañizo Fernández-Roldán J, Pedrosa Durán J, Pozuelos Estrada G. Derivaciones al medio especializado durante 1986 en un centro de salud. Aten Primaria 1988; 5: 85-89.

7. García Benavides F, Cayuela A, Belda J, Gil V. Relación entre la distancia a los servicios de urgencias hospitalarias y su utilización. Rev San Hig Publ 1990; 64: 643-650.

8. Zambrana JL, Díez F, Laynez F, Vázquez J, Cassini L, Gallego F, et al. Calidad del documento de remisión de los pacientes referidos de atención primaria a especializada en un área de salud. Salud Rural 1998; 14 : $65-72$

9. Huertas Zarco I, Pereiró Berenguer I, Sanfélix Genovés J, Rodríguez Moya R. Mejora de la calidad de la hoja de interconsulta a través de la información. Aten Primaria 1996; 17: 317-320.

10. Antón MD, Peña JC, Santos R, Sempere E, Martínez J, Pérula LA. Demanda inadecuada a un servicio de urgencias pediátrico hospitalario: factores implicados. Med Clin (Barc) 1992; 99: 743-746.

11. Delgado Sánchez A. Salud y género en las consultas de atención primaria. Aten Primaria 2001; 27: 75-78.

12. Gómez-Calcerrada Berrocal D, Pérez Flores D, Marset Campos P. Exploraciones y derivaciones en un centro de salud: estudio de los factores asociados. Aten Primaria 1996; 17: 353-357.

13. Cruces Sánchez A, Vaz Leal FJ. Análisis de la influencia de la edad y el género sobre la demanda clínica del paciente. An Psiquiatria 2001; 17(9): 408-412.

14. De la Revilla L, Aranda JM, Luna JD. Influencia de las variables socioeconómicas y de la cultura sanitaria familiar en el uso de las consultas médicas. Aten Primaria 1987; 4: 472-480.

15. Guerrero Fernández M, Alfonso Sánchez JL, Sanchis Noguera B, Prado del Baño MJ. ¿Utilización hospitalaria y ancianidad creciente en el usuario atendido?. Gac Sanit 1992; 6: 62-66.

16. García Olmos L, Gérvas J, García Calleja A, López Ruiz A, Sánchez Rodríguez F, Palomo Cobos L. Episodios de enfermedad atendidos en medicina general/de familia, según medio demográfico (I): morbilidad.

Aten Primaria 1997; 19: 469-476.

17. Palomo Cobos L, García Olmos L, Gérvas J, García Calleja A, López Ruiz A, Sánchez Rodríguez F. Episodios de enfermedad atendidos en medicina general/de familia, según medio demográfico (y II): utilización. Aten Primaria 1997; 20: 82-89.

18. Garrido Cruz I, Montilla Sanz MA, Espejo Rosillo I, Caballero Oliver A, Montero Romero E. Comparación de la derivación de pacientes a un servicio de urgencias hospitalario según medio de procedencia. Aten Primaria 1997; 20: 361-366

19. Rodríguez R, Briso-Montiano R, López M, García J, Blázquez A, Mateos R. Análisis epidemiológico de las consultas de urgencia en atención primaria. Semergen 2001; 27: 561-565.

20. Escobedo F, González Gil L, Salarichs M, Manzano A, López I, Martín JA, et al. Evaluación de las urgencias hospitalarias desde un área básica de salud (ABS). Aten Primaria 1997; 19: 169-175

21. Oterino D, Peiró S, Calvo R, Sutil P, Fernández P, Pérez G, et al. Utilización inadecuada de un servicio de urgencias hospitalario. Una evaluación con criterios explícitos. Gac Sanit 1999; 13: 361-370.

22. Yagüe Hernando AJ, Blanco Ramos MT, Juez Juez AA. Análisis de las derivaciones a las consultas de especialistas realizadas en un centro de salud. Aten Primaria 1991; 8: 472-476.

23. García Gallego JJ, Montero Pérez FJ, Martínez de la Iglesia J, Jiménez López J. Análisis cualitativo de la derivación al segundo nivel. Aten Primaria 1990; 7: 73-74

24. Mate J, Escudero A, Cuberas A, Caballero I, Closas J. Estudio de la solicitud de consulta a especialistas desde la atención primaria. Aten Primaria 1994; 14: 1011

25. Valcayo A, Vives R, Artal F, Eciolaza JM, Parra A, Bernués C, et al. Frecuentación de las consultas de atención primaria por motivos dermatológicos en las zonas básicas de salud de la comarca de Pamplona. Anales Sts San Navarra 1999; 22: 173-179.

26. Strusberg I, Drenkard C, Roqué MH, Gamron S, Barberis G, Onetti CM. Análisis de pacientes ambulatorios que concurren por primera vez al servicio de reumatología en un hospital. Rev Esp Reumatol 2000; 27 : 382-387.

27. Gamero Samino MJ, Bustos Arenas J, Cano Lozano MJ, Gutiérrez Casares JR, Buitrago F. Derivaciones de médicos de atención primaria a una unidad de salud mental. Aten Primaria 1996; 17: 445-448

28. Gracia-Naya M, Marta E, Usón M, Carod J. Estudio epidemiológico descriptivo de una consulta externa de Neurología. Rev Neurol 1996; 24: 633-637.

29. Sáez F, Francia A, Díaz A, Sufrate D, Gómez Ferreras M, Barquin JL Derivaciones desde atención primaria a traumatología: el punto de vista del especialista. Aten Primaria 1997; 20: 570-571.

30. Pellicer C, Fullana J, Cremades MJ, Rivera ML, Perpiñà M. Perfil de una consulta de neumología de ámbito comarcal. Arch Bronconeumol 1996; 32: 447-452. 